\title{
VIEWPOINT
}

\section{Par-4 prevents breast cancer recurrence}

\author{
Nikhil Hebbar ${ }^{1}$, Tripti Shrestha-Bhattarai ${ }^{1}$ and Vivek M Rangnekar ${ }^{1,2,3,4^{*}}$
}

\begin{abstract}
Therapy resistance and disease recurrence are two of the most challenging aspects in breast cancer treatment. A recent article in Cancer Cell makes a significant contribution toward a better understanding of this therapeutic problem by establishing

downregulation of the tumor suppressor Par-4 as the primary determinant of breast cancer recurrence. This viewpoint brings forth the importance of their findings and its implications on future research and therapy.
\end{abstract}

\section{Background}

The primary hurdle in breast cancer therapy is the treatment of tumors which are hormone receptor-negative or triple-negative breast cancers. These tumors tend to be highly resistant to chemotherapy and targeted therapies alike and exhibit a high propensity for recurrence. Once the cancer recurs, the average survival is less than 12 months [1]. Breast cancer is the most commonly diagnosed cancer among women and is second only to lung cancer in terms of cancer deaths [2]. Despite advances in breast cancer research and therapy, over 40,000 deaths in 2013 alone are expected in the US [3].

Breast cancer is characterized by its hormone receptor status that also determines the treatment options that are currently available. Surgery and neoadjuvant chemotherapy have been the mainstay for breast cancer management for a number of years. However, the advent of targeted therapies in combination with chemotherapy has resulted in improved pathological complete response [4]. Currently, a number of targeted therapies such as trastuzumab, pertuzumab, or tamoxifen are designed to kill tumors that are hormone receptor-positive [5]. As a result, patients presenting with positive status for estrogen receptor, progesterone receptor, and/or human epidermal growth factor receptor 2 respond

\footnotetext{
* Correspondence: vmrang01@email.uky.edu

${ }^{1}$ Graduate Center for Toxicology, University of Kentucky, 306 Health Science Research Building, 1095 V.A. Drive, Lexington, KY 40536-0305, USA 'Department of Radiation Medicine, University of Kentucky, 800 Rose Street, Pavilion H, Room C-118, Lexington, KY 40536-0293, USA

Full list of author information is available at the end of the article
}

well to these targeted regimens, showing increased remission and prolonged disease-free survival.

A recent article by Alvarez and colleagues [6] identifies loss of Par- 4 as the fundamental factor leading to breast cancer recurrence. Par-4 is a tumor suppressor that is unique in its ability to selectively induce apoptosis in cancer cells but not in normal cells [7].

\section{The article}

The study by Alvarez and colleagues [6] describes preexisting heterogeneity in a primary tumor population with respect to Par-4 expression and presents a convincing two-step model for breast cancer recurrence in oncogeneaddicted cells. Apoptotic stimuli such as oncogene inhibition or treatment with chemotherapeutic agents cause an acute upregulation in the expression of Par- 4 in these tumor cells, leading to Par-4-induced ZIP-kinasedependent MLC2 phosphorylation, multinucleation, and ultimately apoptosis due to cytokinesis failure. This leads to the initial tumor regression observed with oncogene inhibition or chemotherapy. This study therefore unravels a novel mechanism for Par-4-induced apoptosis in oncogenedependent tumor cells. Pre-existing tumor cells expressing inherently low levels of Par-4 fail to elicit this response, which enables them to survive such apoptotic insults. These cells, therefore, constitute the residual subpopulation that subsequently emerges as recurrent tumor at local or distant sites.

Through a series of well-designed experiments and adept analysis of human breast cancer datasets, the authors demonstrate that downregulation of Par-4 expression is an absolute prerequisite for tumor recurrence and conclude that low Par-4 expression is an independent predictor of decreased recurrence-free survival in women with breast cancer, which corroborates the findings from previous studies $[8,9]$. This is further exemplified by the finding that highly aggressive, estrogen receptor-negative, high-grade (grade 3), and basal-like tumors exhibit extremely low levels of Par-4. 


\section{The viewpoint}

Various other studies have described the loss, downregulation, or inactivation of Par-4 in different tumor scenarios [10]. Oncogenic Ras has been shown to downregulate Par-4 expression through MEK-dependent hypermethylation of the Par-4 promoter [11]; consequently, pancreatic cancers harboring K-ras mutations show diminished expression of Par-4 [12]. In their study, Alvarez and colleagues [6] show that downregulation of Par-4 is 'necessary and sufficient' for breast cancer recurrence, which further underscores the tumor-suppressor function of Par-4. However, the mechanism by which Par-4 is downregulated in recurrent breast tumors is yet to be elucidated. Identifying this mechanism could provide new insights into oncogenic regulation of Par- 4 in the context of breast cancer, making it a promising area for future research.

The article by Alvarez and colleagues [6] makes a valuable contribution toward understanding the mechanisms underlying breast cancer recurrence, thereby opening avenues for new treatment possibilities. An increasing number of women are resorting to prophylactic mastectomy [13], a surgical procedure that can have devastating effects on their physical and emotional well-being. Based on the work by Alvarez and colleagues [6], low Par-4 levels in tumor biopsies can be a powerful prognostic marker for breast cancer recurrence. Using such minimally invasive procedures may provide significant clinical benefit by allowing for more effective therapeutic intervention. Such advances may promote more efficacious personalized therapies and, in so doing, subvert the need for prophylactic mastectomies and reduce patient suffering. The importance of Par-4 as a therapeutic agent can be exploited by using appropriate Par-4 delivery systems, specifically into Par-4deficient cells, thereby restoring chemo-sensitivity and preventing recurrence. The use of such innovative therapeutic approaches is likely to increase overall patient survival and improve the quality of life.

\section{Competing interests}

The authors declare that they have no competing interests.

\section{Acknowledgments}

This work was supported by National Cancer Institute/National Institutes of Health grant CA060872 and Kentucky Lung Cancer Research grant (to VMR).

\footnotetext{
Author details

${ }^{1}$ Graduate Center for Toxicology, University of Kentucky, 306 Health Science Research Building, 1095 V.A. Drive, Lexington, KY 40536-0305, USA.

${ }^{2}$ Department of Radiation Medicine, University of Kentucky, 800 Rose Street Pavilion H, Room C-118, Lexington, KY 40536-0293, USA. ${ }^{3}$ Departments of Microbiology, Immunology and Molecular Genetics, University of Kentucky, 800 Rose Street, MS-415, Lexington, KY 40536-0298, USA. ${ }^{4}$ L. P. Markey Cancer Center, University of Kentucky, 800 Rose Street, Lexington, KY 20537-0293, USA.
}

\section{References}

1. Guarneri V, Dieci MV, Conte P: Relapsed triple-negative breast cancer: challenges and treatment strategies. Drugs 2013, 73:1257-1265.

2. Theriault RL, Carlson RW, Allred C, Anderson BO, Burstein HJ, Edge SB, Farrar WB, Forero A, Giordano SH, Goldstein L, Gradishar WJ, Hayes DF, Hudis CA, Isakoff SJ, Ljung BM, Mankoff DA, Marcom PK, Mayer IA, McCormick B, Pierce L, Reed EC, Schwartzberg LS, Smith ML, Soliman H, Somlo G, Ward JH, Wolff AC, Zellars R, Shead DA, Kumar R: Breast cancer, version 3.2013. J Natl Compr Canc Netw 2013, 11:753-761.

3. Siegel R, Naishadham D, Jemal A: Cancer statistics, 2013. CA Cancer J Clin 2013, 63:11-30.

4. Rea D, Tomlins A, Francis A: Time to stop operating on breast cancer patients with pathological complete response? Eur J Surg Oncol 2013, 39:924-930.

5. Pritchard Kl: Endocrine therapy: is the first generation of targeted drugs the last? J Intern Med 2013, 274:144-152.

6. Alvarez JV, Pan TC, Ruth J, Feng Y, Zhou A, Pant D, Grimley JS, Wandless TJ, Demichele A: I-SPY 1 TRIAL Investigators, Chodosh LA: Par-4 downregulation promotes breast cancer recurrence by preventing multinucleation following targeted therapy. Cancer Cell 2013, 24:30-44.

7. Hebbar N, Wang C, Rangnekar VM: Mechanisms of apoptosis by the tumor suppressor Par-4. J Cell Physiol 2012, 227:3715-3721.

8. Méndez-López LF, Zapata-Benavides P, Zavala-Pompa A, Aguado-Barrera ME, Pacheco-Calleros J, Rodríguez-Padilla C, Cerda-Flores RM, Cortés-Gutiérrez El, Dávila-Rodríguez MI: Immunohistochemical analysis of prostate apoptosis response-4 (Par-4) in Mexican women with breast cancer: a preliminary study. Arch Med Res 2010, 41:261-268.

9. Nagai MA, Gerhard R, Salaorni S, Fregnani JH, Nonogaki S, Netto MM, Soares FA: Down-regulation of the candidate tumor suppressor gene PAR-4 is associated with poor prognosis in breast cancer. Int J Oncol 2010, 37:41-49.

10. Shrestha-Bhattarai T, Rangnekar VM: Cancer-selective apoptotic effects of extracellular and intracellular Par-4. Oncogene 2010, 29:3873-3880.

11. Pruitt K, Ulku AS, Frantz K, Rojas RJ, Muniz-Medina VM, Rangnekar VM, Der CJ, Shields JM: Ras-mediated loss of the pro-apoptotic response protein Par- 4 is mediated by DNA hypermethylation through Raf-independent and Rafdependent signaling cascades in epithelial cells. J Biol Chem 2005, 280:23363-23370.

12. Ahmed MM, Sheldon D, Fruitwala MA, Venkatasubbarao K, Lee EY, Gupta S, Wood C, Mohiuddin M, Strodel WE: Downregulation of PAR-4, a pro-apoptotic gene, in pancreatic tumors harboring K-ras mutation. Int J Cancer 2008, 122:63-70.

13. Lostumbo L, Carbine NE, Wallace J: Prophylactic mastectomy for the prevention of breast cancer. Cochrane Database Syst Rev 2010, 11, CD002748.

$10.1186 /$ bcr3562

Cite this article as: Hebbar et al:: Par-4 prevents breast cancer recurrence. Breast Cancer Research 2013, 15:314 